\title{
PERFORMANCE OF THE EXTERNAL MUON IDENTIFIER FOR THE BIG EUROPEAN BUBBLE CHAMBER
}

\author{
R. BEUSELINCK*, R. C. A. BROWN, E. CHESI, P. M. FERRAN, \\ H. FOETH, A. GILGRASS**, H. J. HILKE*** D. JACOBS, \\ P. LAZEYRAS, Y. SAQUIN ${ }^{+}$and M. E. J. WIGMANS ${ }^{++}$
}

CERN, Genera, Switzerland

Received 6 March 1978

\begin{abstract}
Muon identification in experiments with BEBC is obtained by a combination of two planes of multiwire proportional chambers and a hadron absorber of iron (thickness up to $210 \mathrm{~cm}$ ). The detector planes cover 18 and $150 \mathrm{~m}^{2}$, respectively, and contain some 85000 sense wires and $200 n 0$ electronic channels. This system has successfully operated in $v$ and $\bar{v}$ experiments, the matin part of it for a year. Under most severe background conditions up to 600 particles were registered in a $2 \mathrm{~ms}$ beam spill. The performance of the chambers, the fast electronics and the on-line data handling system is described. Ar/ $\mathrm{CO}_{2}$ mixtures have proved to be very reliable. A short account is given on the next improvenent stage.
\end{abstract}

\section{Introduction}

Most of the experiments with BEBC at SPS energies require particle identification of the tracks leaving the bubble chamber. With the External Muon Identifier (EMI) the muon identification can

* Visitor from Imperial College, London, U.K.

** Visitor from the University of Oxford, U.K.

*** On leave at Lawrence Berkeley Lab., Berkeley, U.S.A.

- Visitor from C.E.N. Saclay, France.

- Visitor from N.I.K.H.E.F., Amsterdam, the Netherlands. be achieved by reconstruction. Through some ten hadron interaction lengths of absorber only muons will remain close to the track when extrapolated through the outer wire chamber plane. The aim was to keep the background from pion punchthrough down to $<1 \%$ and the $\mu$ background from $\pi$ decay down to a few per thousand.

A major problem is the high background level in the wide-band (anti)-neutrino beams: up to 600

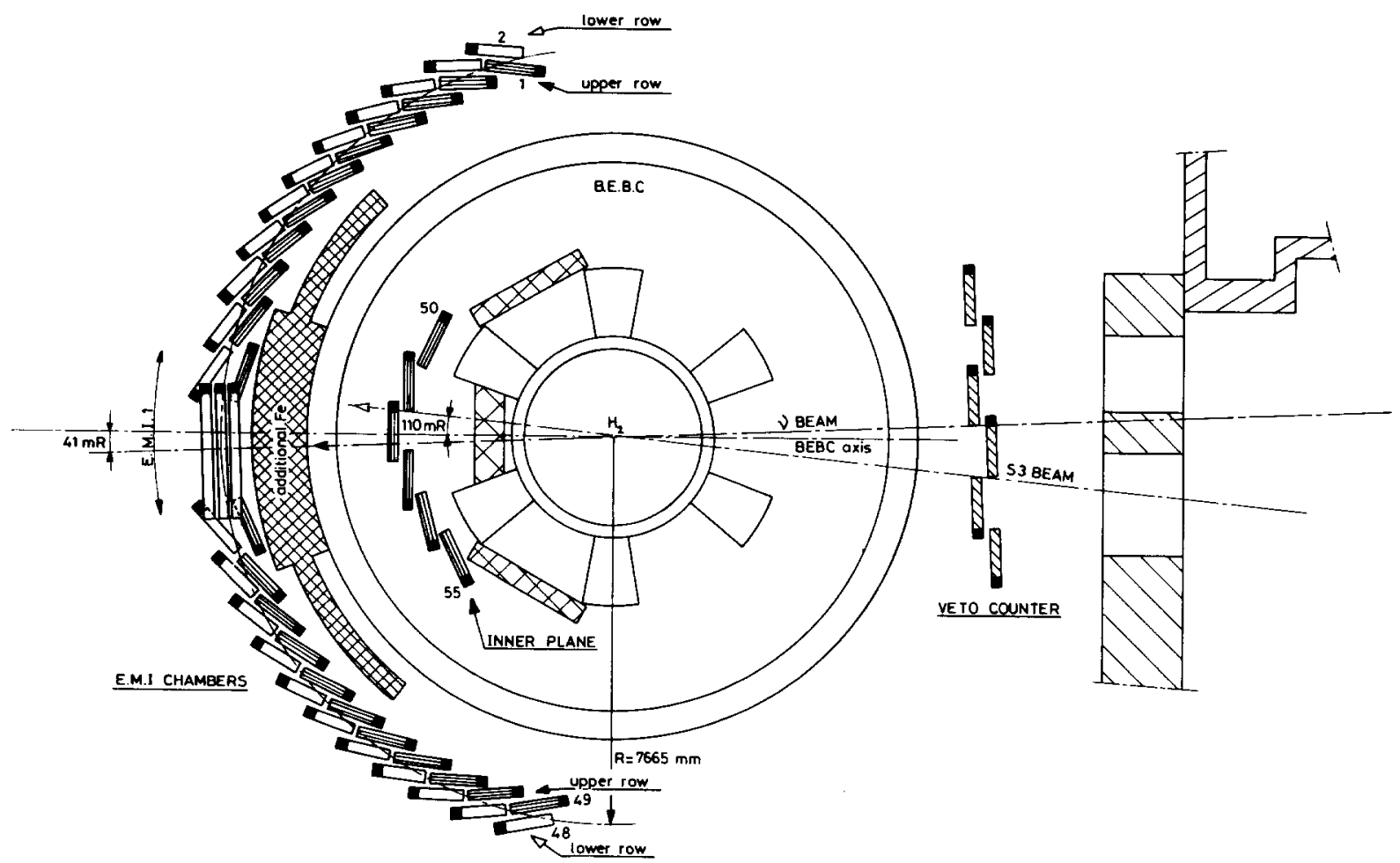

Fig. 1. General arrangement of the EMI. 
particles over the whole outer plane, and 60 in a single chamber were recorded during a $2 \mathrm{~ms}$ spill of $5 \times 10^{12}$ protons at $350 \mathrm{GeV} / \mathrm{c}$. In principle, up to 1023 events per beam spill can be accepted by the electronics for the total EMI and up to 79 for single chambers. The event time information helps to avoid ambiguities in the reconstruction of the events (the time resolution is $\sim 0.5 \mu \mathrm{s}$ ).

A detailed description of the system is given elsewhere').

\section{General lay-out}

A schematic view of the arrangement of the two detector planes and the absorber is given in fig. 1 . Fig. 2 shows the outer plane as it looks in reality. The inner detector plane is situated at $\sim 4 \mathrm{~m}$ from the centre of $\mathrm{BEBC}$ and covers the solid angle between $-43^{\circ}$ and $+35^{\circ}$ horizontally and $-18^{\circ}$ and $+18^{\circ}$ vertically. The outer plane at approximately $7.6 \mathrm{~m}$ from the BEBC centre stretches from $-80^{\circ}$ to $+80^{\circ}(\sim 24 \mathrm{~m})$ horizontally and from $-20^{\circ}$ to $+20^{\circ}$ vertically $(\sim 6 \mathrm{~m})$. The absorber upstream from the inner plane $(50 \mathrm{~cm} \mathrm{~Pb}$ in the forward cone, 2 to 3 hadron interaction lengths of $\mathrm{Fe}$ and coil materials eisewhere) mainly aims at a reduction of the $\mu$ background produced by $\pi$ decay.

The detector planes consist of chamber modules $3 \times 1 \mathrm{~m}$. Each module gives three hit coordinates, two from independent sense wire planes and one from cathode strips read-out. The channel width varies from $8 \mathrm{~mm}$ in the forward cone to $32 \mathrm{~mm}$ at large angles in the outer plane. The inner plane consists of modules with a channel width of $32 \mathrm{~mm}$.

\section{The operation of the system}

\subsection{General}

The complete outer plane has been used in experiments for nine months; during the last six of these, the inner plane was available as well. Most of the experiments were performed with neutrino

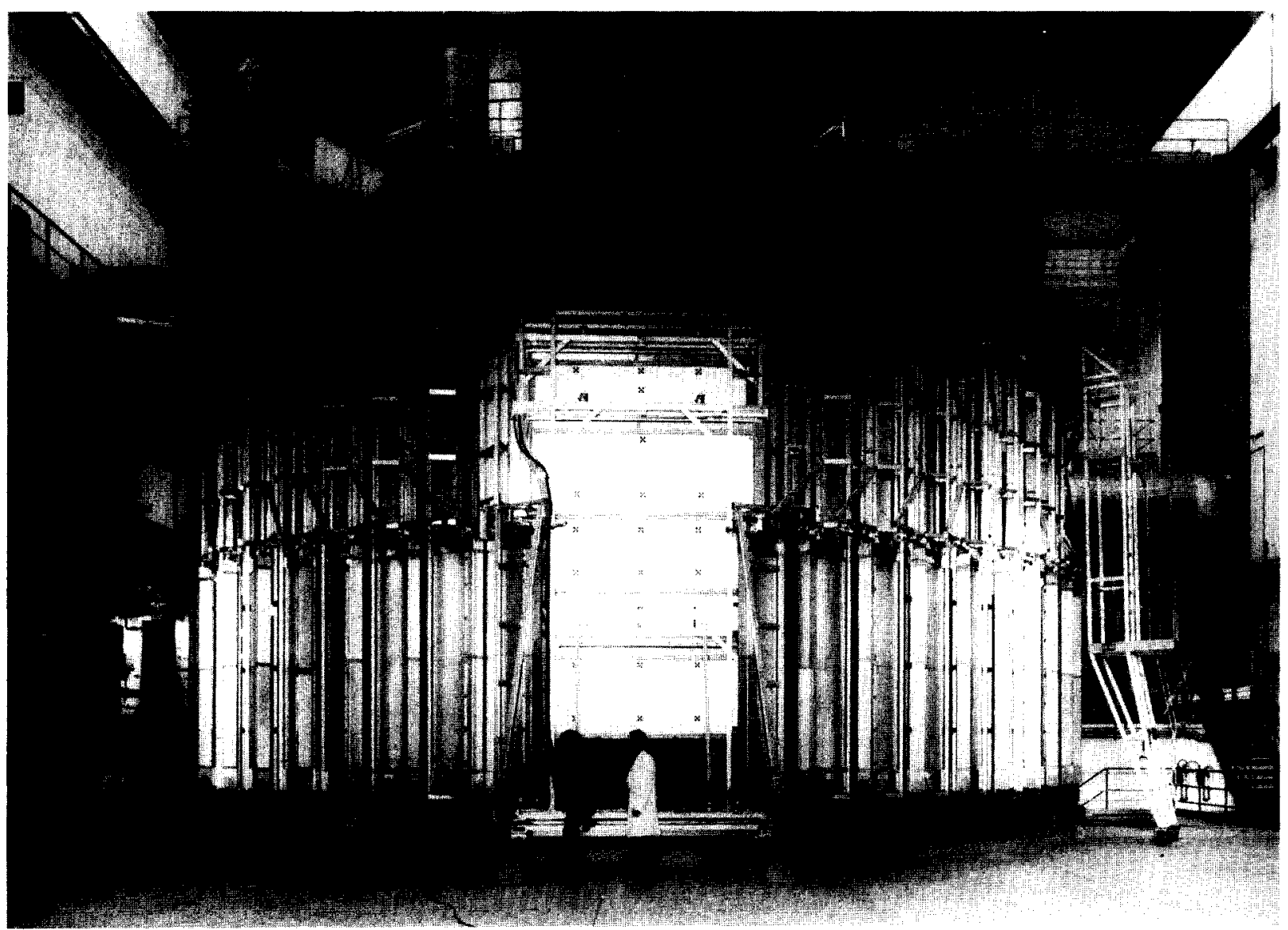

Fig. 2. The EMI of BEBC (outer plane). 
or anti-neutrino beams with spill times of either $23 \mu \mathrm{s}$ or $2 \mathrm{~ms}$. In wide-band beams the severe beam-associated $\mu$ background level was limiting the maximum beam energy for a given beam intensity. For a wide-band beam formed during a $2 \mathrm{~ms}$ spill by $5 \times 10^{12}$ protons on the target at $350 \mathrm{GeV} / \mathrm{c}$, about 600 tracks were recorded by the EMI in some 300 time slots. Some chambers recorded up to 100 tracks in 50 time slots per spill.

The overall single plane efficiency for the offline $\mu$ reconstruction is at present $\sim 98 \%$. This figure was obtained from the analysis of background muon tracks with a momentum $>5 \mathrm{GeV} / \mathrm{c}$ and data of the one plane EMI.

\subsection{THE ELECTRONICS}

\subsubsection{Introduction}

The principles of the electronics system for the recording of events have been described extensively before'). Basically, each electronic channel (group of sense wires or cathode strips) is connect- ed to a shift register. Whenever an event occurs, the hit pattern is clocked into all shift registers. The speed of the shift registers $(2 \mathrm{MHz})$ determines the time resolution of the system. For each event, the time and the complete hit pattern (chamber and channel numbers) are written on tape.

From an electronic point of view, the two detector planes of the EMI are regarded as one detector. The system is self-triggered by hits in either of these two planes. The correlation between hits in the two planes is done off-line by event time analysis in combination with track extrapolation from the $B E B C$ picture.

\subsubsection{The synchronization with the SPS}

The data acquisition cycle is governed by trains of prepulses which precede the extractions of the SPS proton beam. Up to now, a $-600 \mathrm{~ms}$ signal serves as an external interrupt to reset the computer and the CAMAC logic, while a $-50 \mu \mathrm{s}$ pre-



Fig. 3. The on-line computer system. 
pulse is used to open the external gate for the data taking during the extraction of protons for the $(\bar{v})$ beam. One pulse is used to strobe off the high $\mu$ background during the slow extraction of the $200 \mathrm{GeV}$ protons for the counter experiments in the West Hall. This is necessary when the behaviour of individual modules is tested with cosmic rays.

When $\mathrm{BEBC}$ is operating in the double pulsing mode, i.e. when during an SPS cycle besides a ( $v$ ) picture, a picture is also taken of a hadron beam $\sim 3 \mathrm{~s}$ before the ' $\bar{v}$ ' extraction, EMI data can be taken for both pictures, provided that appropriate prepulses are associated with the extraction for the hadron beam. In this case the prepulse is also used to generate a hadron identifier signal in order to be able to distinguish between the ' $v$ ' and hadron data on tape. This data taking mode has

\section{EMI ONLINE SOFTWARE}

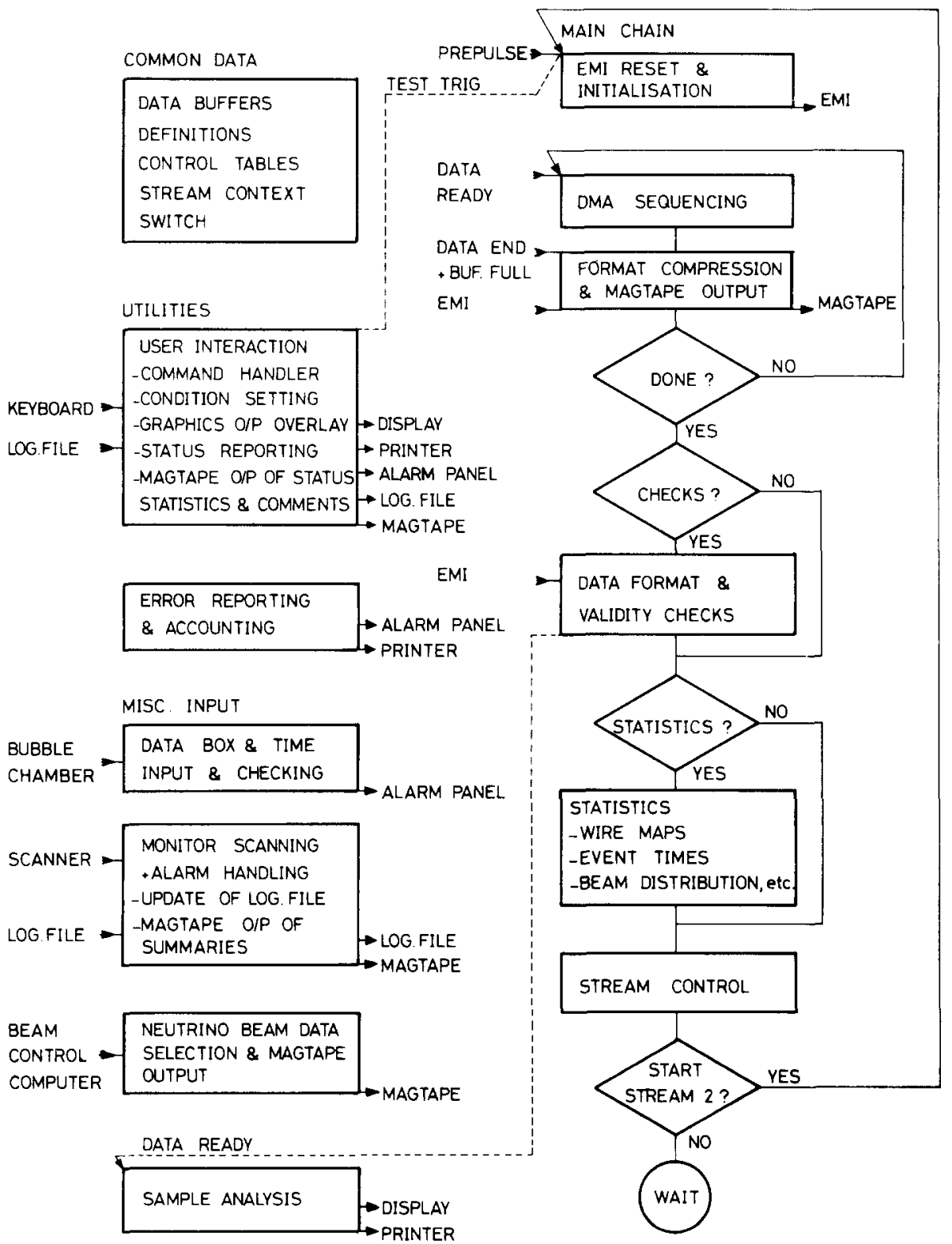

Fig. 4. The EMI software outline. 
been tried successfully. Also triple pulsing, i.e. with hadron pictures taken at the beginning and the end of the $200 \mathrm{GeV}$ flat top, does not cause fundamental difficulties for the EMI provided that the first extraction for the hadron beam is of the fast type ( $5 \mu \mathrm{s}$ duration), so that cosmic ray background is negligible.

\subsection{Data aCQuisition AND testing}

\subsubsection{Program structure}

Fig. 3 shows the configuration of the on-line computer. The NORD-10 and its operating system SINTRAN-III, provide a multi-tasking, virtual memory environment and the EMI software has been written to make use of these features. It consists of a package of some 14 real time tasks, mostly written in FORTRAN, occupying a total virtual memory space of $130 \mathrm{kw}$. Of this total, about $12 \mathrm{kw}$ is locked in memory and the rest is paged on demand. The package is self-contained and is automatically initialized by push-button or computer restart. All users' dialogue passes via the package using an application-oriented command structure, and he need not become familiar with the intricacies of the SINTRAN-III operating sys- tem. The package is shown in simplified form in fig. 4. The tasks communicate with each other and have access to common data buffers via a SINTRAN-III construct which allows them to share a segment in virtual memory space.

\subsubsection{Data acquisition}

The two main design decisions for the data acquisition were to decide how much data reduction should be attempted and what should be the magnetic tape format. For accurate point reconstruction it is necessary to take into account the expected tilt of candidate tracks measured in BEBC and so on-line reduction was restricted to format compression by grouping of neighbouring channels into clusters. The resultant compression is $\sim 30 \%$. The tape format was chosen in collaboration with other groups at CERN [2] and is based on a fixed physical record length of 1890 16-bit words, giving good tape utilization. The logical records (e.g. the EMI data from one spill) are entirely decoupled from the physical ones and are accessed via a system of pointers and word counts. The data is checked for formal validity as it is output and may also be scanned in order to update histograms as detailed below.

EMI CYCLING IN 2-STREAM MODE

(1= Beam data, 2 = Tests)
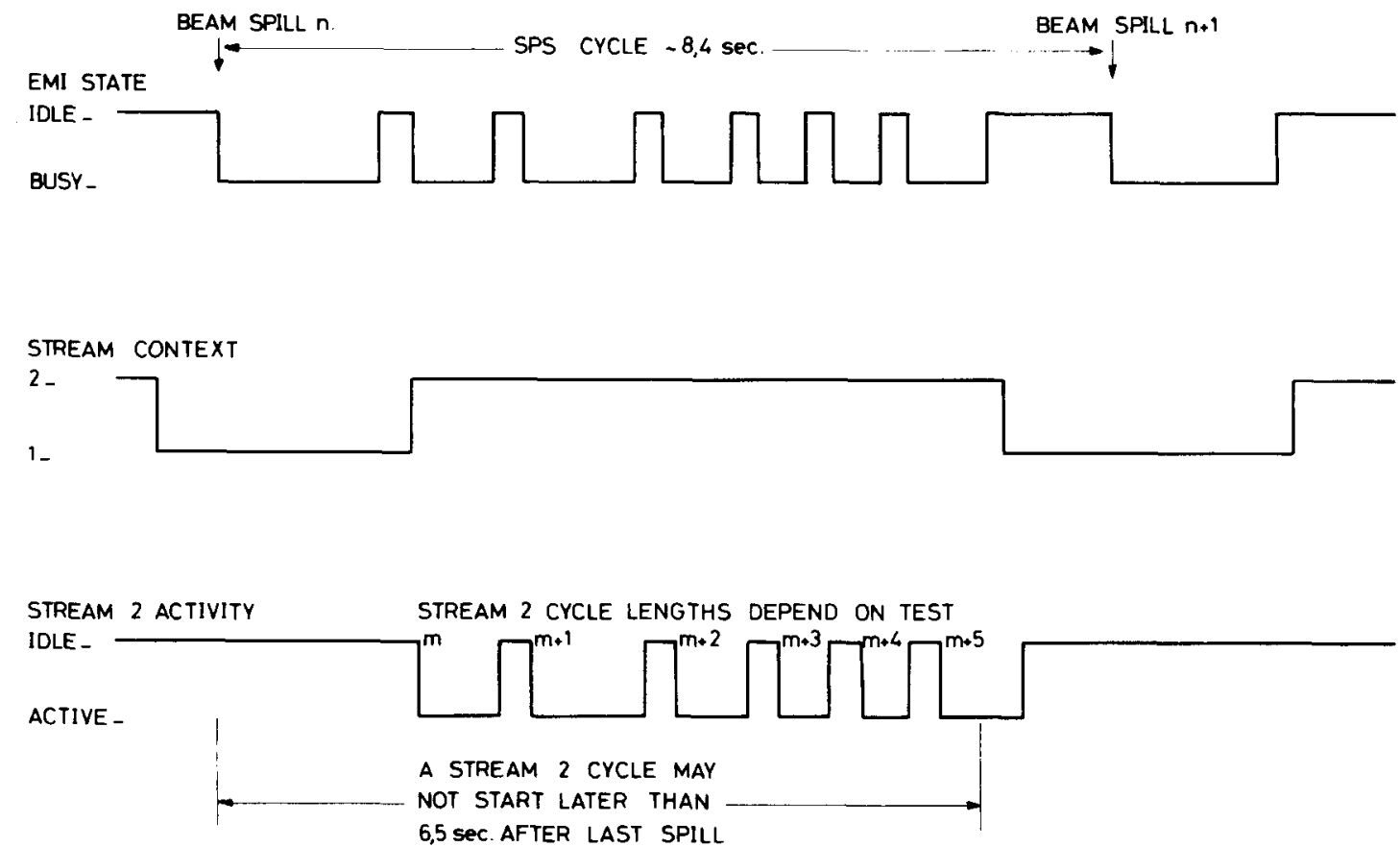

Fig. 5. The EMI cycling in two-stream mode. 


\subsubsection{EMI performance testing}

Positive identification of muons requires that the EMI chambers operate at very high efficiency and so the on-line checking of this is most important. It would be very inconvenient to do this testing on data acquired during the beam spill in view of its extremely uneven space distribution and the low rate of hit accumulation in any given chamber. For this reason, mechanisms were included in hardware and software to allow the data acquisition chain also to operate between beam spills, collecting data from cosmic rays or doing digital tests. The strategy for this is shown in fig. 5 . The data taking proceeds in two "streams". Stream I takes data synchronized with the beam spill. As soon as the processing of these data is complete, the computer switches the conditions or "context" of the EMI to those needed for testing (stream 2) and executes as many cycles of test data taking as possible before the next spill arrives. Experience has shown that the most commonly used tests during production runs are:

a) Verification of chamber efficiencies by examining wire maps. It is possible to automatically step through all chamber modules applying this test.

b) Comparison of hit rates in chamber modules. In stream 1, this gives the spacial profile of the beam. and in stream 2 a rapid overall measure of chamber efficiencies

c) Verification of beam time profile by histogramming the EMI event times.

In addition, tests exist to cover more specialized needs; testing of shift registers with digital data, pulsing chamber cathodes to induce signals on all channels, testing data cables, etc.

\subsubsection{Monitoring}

The large number of power supplies in the EMI and its status, as a facility to be used by many visiting groups, dictated automatic scanning of voltages by the computer. At present, some 500 points are measured at a normal rate of one per second. They are compared against acceptable limits and warnings are given as necessary. In addition, more serious failures such as HT overcurrent alarm cause interrupts, and fast scans are made to identify the source.

\subsubsection{Data bases}

The running of the EMI is facilitated by the use of several data-base files updated and accessed by programs of the package:

a) Chamber definition and status. Amongst other things, this contains a list of channels known to be bad. When wire maps are accumulated, bad channels are identified. If they are not already in the list, the user is informed.

b) Chamber performance log. This holds, for each chamber, the measured values of efficiency, cluster width etc., the available space being reused circularly so that the most recent values are available.

c) Monitor definitions and limits.

d) Monitoring $\log$. This operates in a similar fashion to the performance log. The monitoring lask enters measured voltages, whenever they differ by more than a specified amount from the previous measurement.

\subsubsection{Development and secondary experimems}

From the start, the software has undergone rapid evolution and the ability confered by SIN. TRAN-III, to do development and install modifications while in production, has proved most useful. The rate of modification is now decreasing and the system will soon be virtually stable. From time to time additional detector arrays will be added to BEBC for specific experiments and read out via the EMI system. The first of them is already in operation and has proved simple to interface to the EMI software as a set of independent tasks activated from a small number of hooks in the main chain.

\subsection{TIIE GAS SYSTEM}

The multiwire proportional chambers operate with an $\mathrm{Ar} / \mathrm{CO}_{2}$ mixture of $70 / 30$ volume per cent. These gases were chosen on the basis of security (non-inflammable), easy control and low cost. Standard $\mathrm{Ar}(>99.995 \%)$ and $\mathrm{CO}_{2}(>99.9 \%$ purity) are mixed with a flow controlled to better than $1 \%$ and passed through a two micron filter. The average flow per chamber was usually $200 \mathrm{ml} / \mathrm{min}$.

So far, no deterioration of the chamber performance has been observed. Not a single wire (out of some 85000 ) broke due to a discharge.

\subsection{THE SAFETY SYSTEM}

The whole of the EMI chamber installation is in a hydrogen zone, requiring precautions to prevent hydrogen gas from coming into contact with live 
voltages in the chamber or power supply electronics. The chamber power supplies are pressurized with compressed air. The chambers of the outer plane are fitted with air-tight covers over the electronics, and are also pressurized, using the exhaust of the chamber gas. Failure of the pressurization is detected by pressure switches in an intrinsically safe relay circuit, which cuts all voltages in the chamber concerned. Since the bias voltage from the memory buffer line receivers appears in the chambers, it was considered necessary to cut off supplies to the appropriate buffer crates as well.

Chambers in the inner plane are operated in the inert nitrogen atmosphere which fills the BEBC shield during hydrogen running, and additional pressurization was not required.

Detection of hydrogen in the BEBC atmosphere, or either hydrogen or oxygen within the BEBC shield, results in supplies to the entire EMI system being cut-off. The false alarm rate is about one incident per month: the system can be brought into operation again within a few mi-

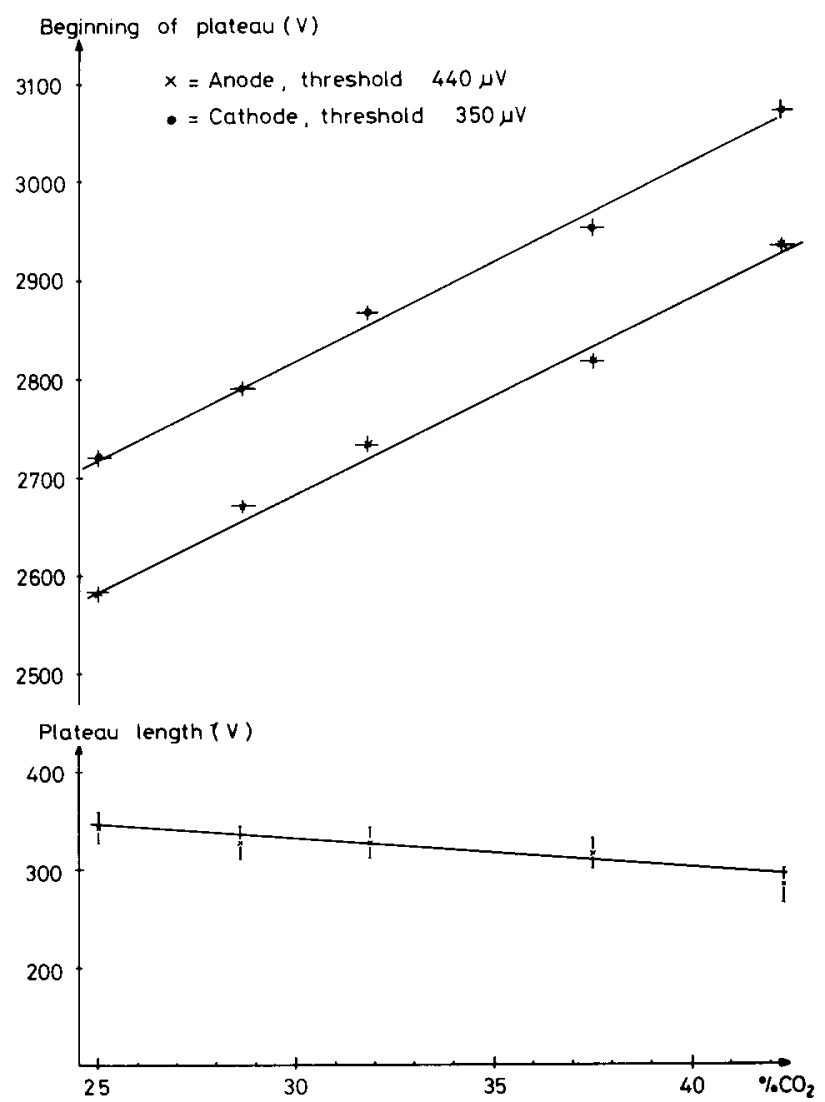

Fig. 6. The dependence of plateau characteristics on the gas composition. nutes. The computer, which is not in a hydrogen zone, is not cut-off by the safety system.

\subsection{The CHAMBERS}

\subsubsection{Electronic threshold and noise}

Despite the large surface area of the detector the system proved to be rather noisefree thanks to careful screening and grounding. None of the chambers showed a significant increase in counting rate down to $150 \mu \mathrm{V}$ input threshold (on preamplifiers with an input impedance of $1.8 \mathrm{k} \Omega$ and a bandwidth of $6 \mathrm{MHz}$ ). The value of $150 \mu \mathrm{V}$ is an average as the threshold varies quite substantially amongst the preamplifiers for a given bias, which is common to a full chamber. The standard threshold is set to about $160 \mu \mathrm{V}$ on the cathode strips and $260 \mu \mathrm{V}$ on the anode wires.

\subsubsection{Chamber plateaux and the choice of working points}

The chamber plateau, i.e. the high voltage region where the detection is fully efficient without discharges, depends strongly on the gas composition and the signal thresholds. This is illustrated in figs. 6 and 7. Fig. 6, which emphasizes the need to keep the gas mixture constant, shows that a change of the $\mathrm{CO}_{2}$ content causes a general plateau shift and does not influence the length of the plateau to a great extent, whereas the signal

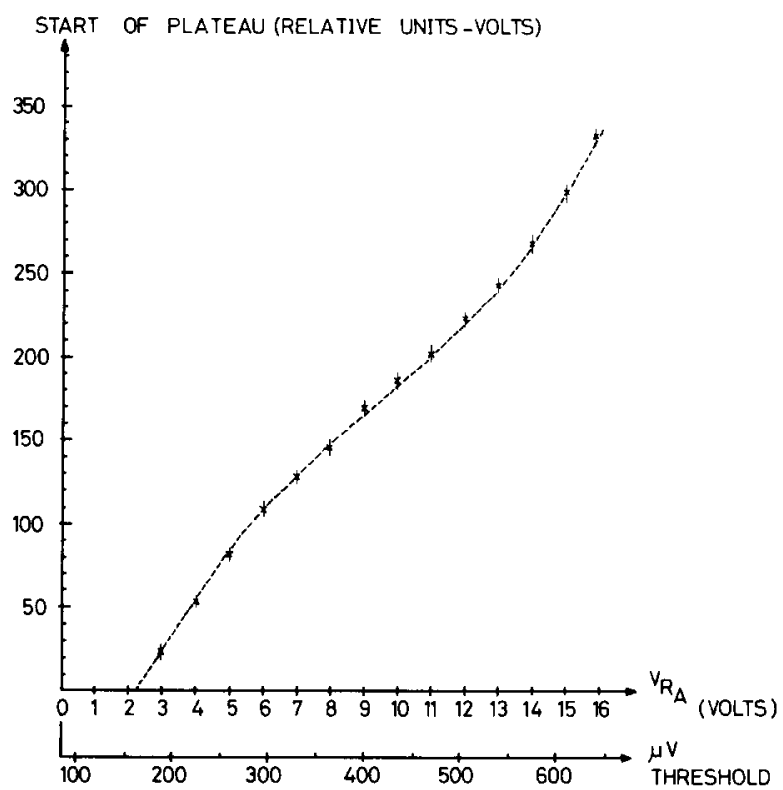

Fig. 7. The dependence of plateau characteristics on the signal thresholds. 
thresholds only influence the beginning, and therefore the length, of the plateau (fig. 7). However, the gas mixture and the signal bias being the same for all modules, the differences between the plateau values of the various chambers turned out to be small $(<100 \mathrm{~V})$.

The working voltage of each chamber was chosen such that the efficiency of the cathode was $\geqslant 99.0^{\circ}$. For our particular choice of thresholds (section 3.6.1), this meant that the anodes reached full efficiency at $\sim 100-150 \mathrm{~V}$ below this value. The working points of all modules were found in the region $(2.85 \pm 0.05) \mathrm{kV}$.

\subsubsection{Cathode efficiency}

The values for the cathode efficiency were obtained using cosmic radiation and triggering on the coincidence signal from both anodes. Of course, these anodes should be fully efficient to make the obtained values meaningful.

The variation of cathode efficiency with high tension is shown in fig. 8. Presented are the results for chambers with cathode strip widths of 16 and $32 \mathrm{~mm}$, respectively. The two chamber types show substantially the same dependence. Fig. 9 indicates the high tension required to obtain a fixed cathode efficiency (of 99\%) as a function of the electronic threshold. Again, no significant dependence on the width of the cathode strip was observed.

\subsubsection{Cluster width}

The number of adjacent electronic channels touched in a single particle crossing will be called cluster width. The cluster width mainly depends on the angle between the particle trajectory and the orientation of the wires or cathode strips. For the latter, moreover, it depends on the amplitude

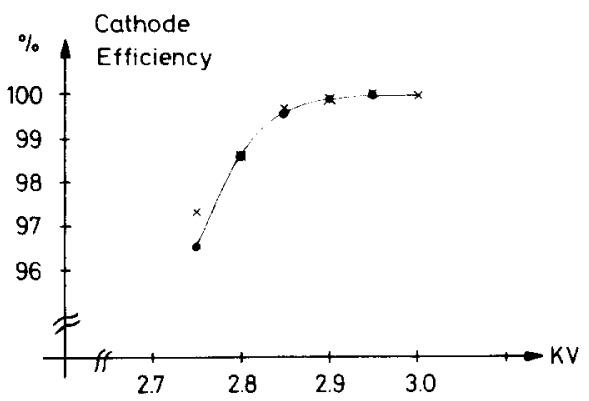

Fig. 8. Cathode efficiency as a function of HT for cathode strips of $32 \mathrm{~mm}(x)$ and $16 \mathrm{~mm}(-)$ wide. Electronic threshold is $160 \mu \mathrm{V}$ for the cathode. of the avalanche (due to pulse formation by induced charges with a finite threshold).

To cope with the requirements for space resolution, three types of chambers were built, which differ in the number of anode wires (and cathode strips) interconnected on the printed board. Thus, the high medium and low resolution chambers have $2(1), 4(1)$ and $8(2)$ anode wires (cathode strips) respectively, coupled to one electronic channel.

The cluster width at fixed high tension, is obviously different for chambers with different groupings, as can be seen in fig. 10. The results shown in this figure were obtained from measurements on cosmic radiation triggering on coincidences between the two anode planes of a chamber. The mean cluster width on the cathodes strongly depends on the high tension. For the anodes this dependence is weak, as may be expected. Results for the high resolution chambers are not included, as they have a different orientation and are much closer to the iron absorber.

For the beam associated particles similar results were obtained, except that the values for the mean cluster width were found to be much closer to 1 . This is explained by the fact that all these particles traverse the detector more or less perpendicularly.

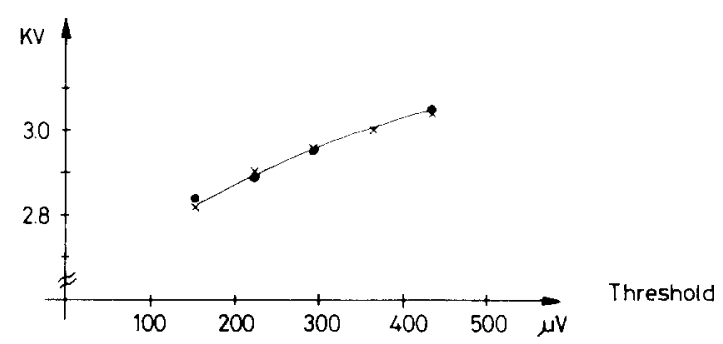

Fig. 9. IIT required to obtain a cathode efficiency of $99 \%$ as a function of electronic threshold. Cathode strips are $32 \mathrm{~mm}$ $(x)$ or $16 \mathrm{~mm}$ (O) wide.

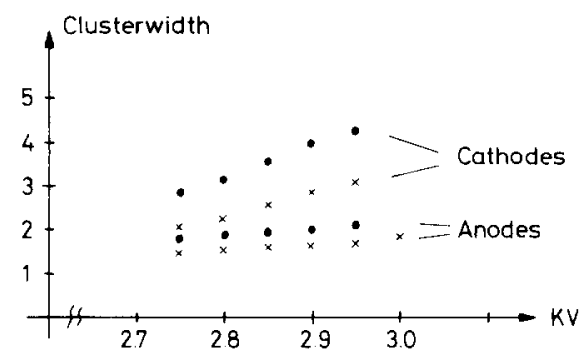

Fig. 10. Cluster widths for cathodes and anodes as a function of HT measured for cosmic radiation on a low $(x)$ and a medium (-) resolution chamber. Electronic thresholds are 160 and $370 \mu \mathrm{V}$ for the cathodes and anodes, respectively, 


\subsubsection{Long-term performance}

(a) The chambers. So far, no long term change in the threshold for full efficiency, the plateau length or the untriggered counting rates has been observed. Over long periods, all chambers have been operated without a single discharge on the 85000 wires. This is illustrated by fig. 11 , which shows the free running count rates for cosmic radiation triggering on either of the two wire planes in any chamber. The small variations that occur for the modules in the centre of the outer plane are due to the varying distance from the iron absorber. This absorber also causes the reduction of $>40 \%$ for the counting rates in the inner detector plane.

Small short-term variations in the chamber characteristics were observed. They correspond to voltage changes of $<30 \mathrm{~V}$, and are mainly caused by instabilities in the gas mixture. So far, four wires have broken in the installed system over a one year's period. All four had been badly soldered at the very end of the soldering pad.

(b) The electronics. As a consequence of the operation within the BEBC hydrogen safety zone it is difficult to replace any electronic equipment which is mounted on the chambers. For this reason, the electronics at the chambers has been kept to a minimum, consisting of an amplifier, a discriminator and a line driver for each channel. Whenever one of these components fails it cannot be replaced until a convenient shut-down and the corresponding channel is lost during this time. The storage electronics, the CAMAC logic, the timing electronics for the synchronization with the SPS, and the NORD-10 computer are located in a control room outside the hydrogen safety zone and problems occurring in this region can always be cured without access difficulties.

The main part of the problems that occur is caused by bad contacts or dead components. If this happens in the storage electronics or the connections of these with the chambers, it may cause a loss of individual channels. If it occurs in the logic part, it may cause a complete loss of data until the problem is cured, because a serial read-out system is used.

In spite of the tremendous number of components that form the EMI electronics (in total 18048 channels) the number of problems which occur is modest. During the first year of operation, it was possible to keep the number of lost channels below $0.6 \%$. Fig. 12 shows the integrated number of dead amplifier cards during this period. It appears
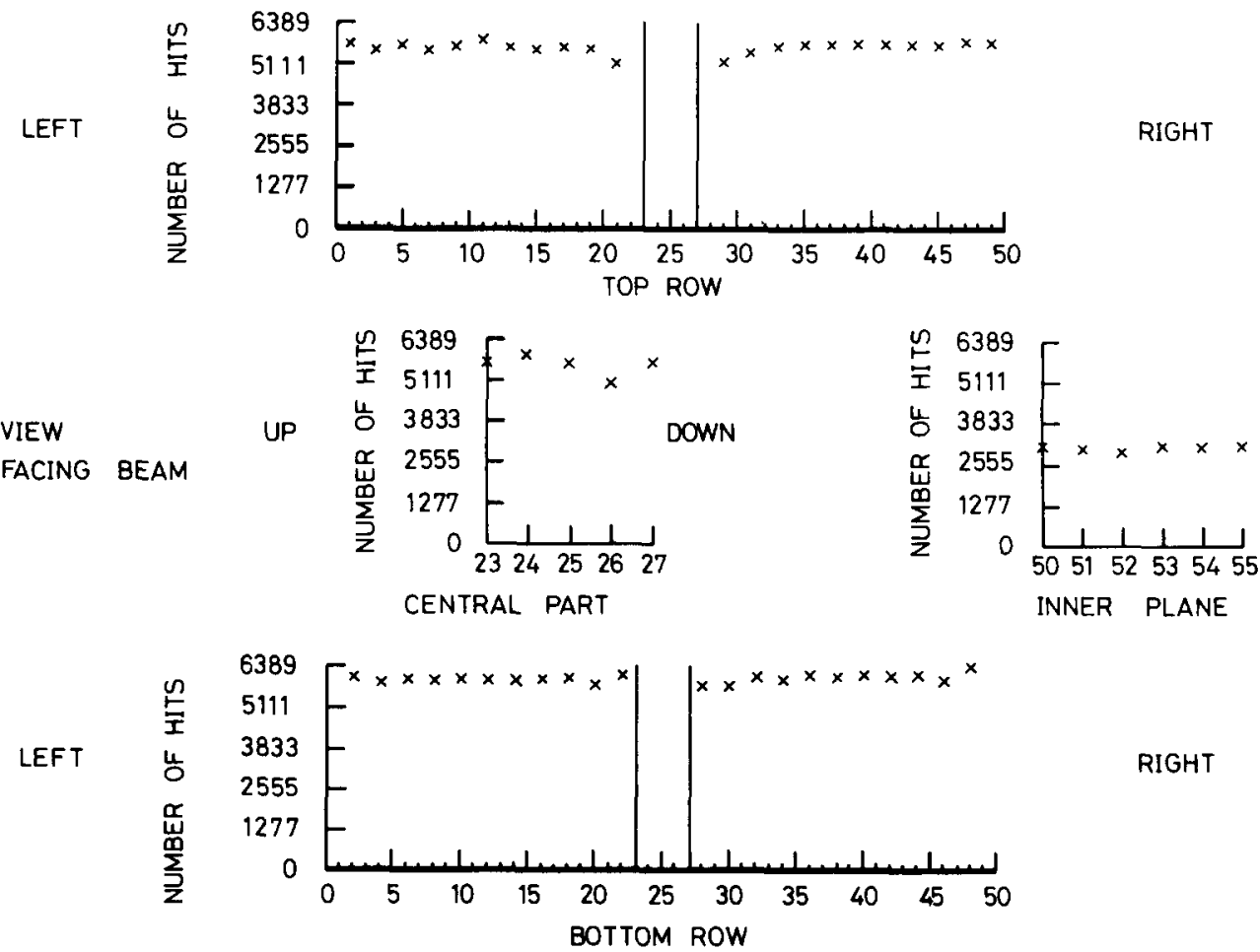

Fig. 11. Single rates of the chambers from cosmic radiation (see text). 


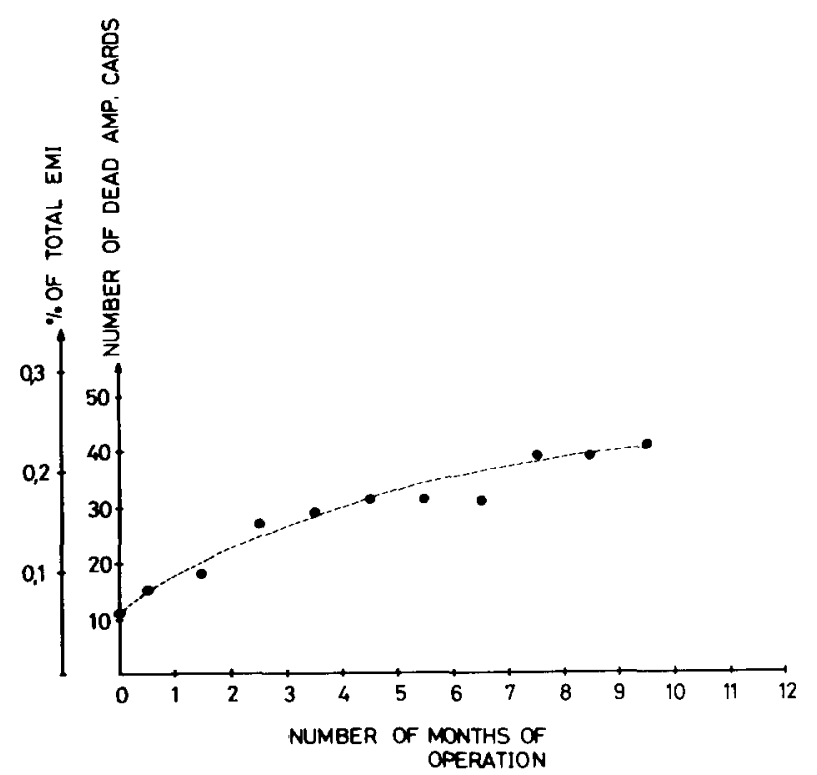

Fig. 12. The drop-out of amplifier cards (integrated numbers).

that they account for $30-50 \%$ of the total loss of channels.

The loss of beam data due to problems with the logic is $<1 \%$, whereas the total portion of BEBC pictures for which no EMI information exists for whatever reason, amounted to $5 \%$ in 1977.

\section{Developments envisaged for near future}

The following projects to improve the system should be mentioned:

a) The construction of a veto-counter upstream from BEBC (fig. 1) with dimensions $6 \times 3 \mathrm{~m}$ consisting of standard low resolution EMI chambers. This device will facilitate the recognition of the beam-associated muon part of the background in the EMI.

b) The development of an optional possibility to trigger on coincidence, between the inner and outer EMI detector planes, in order to be able to run at intensities which would otherwise exceed the buffer capacity.

c) The equipment of a second cathode read-out for the inner plane chambers in order to keep a possibility for point reconstruction in case a wire breaks in one of these very inaccessible chambers.

The authors wish to thank the technicians: C. Brand, M. Brunet, G. di Tore and their collaborators for their help on modifications and repairs. P. Cennini, S. Cairanti, D. Marland and H. Masuch developed miscellaneous electronic equipment. We are grateful for the friendly collaboration of the BEBC crews and the neutrino beam group, as well as for the continuous effort put in by the various user groups during the runs. The feedback provided by the data analyzing groups has been of the greatest help in debugging the system.

\section{References}

1) C. Brand et al., Nucl. Instr. and Meth. 136 (1976) 485

2) J. Ogilvie, Nord Information Notes. CERN EP-Division, ND $77-1 / 1$ 